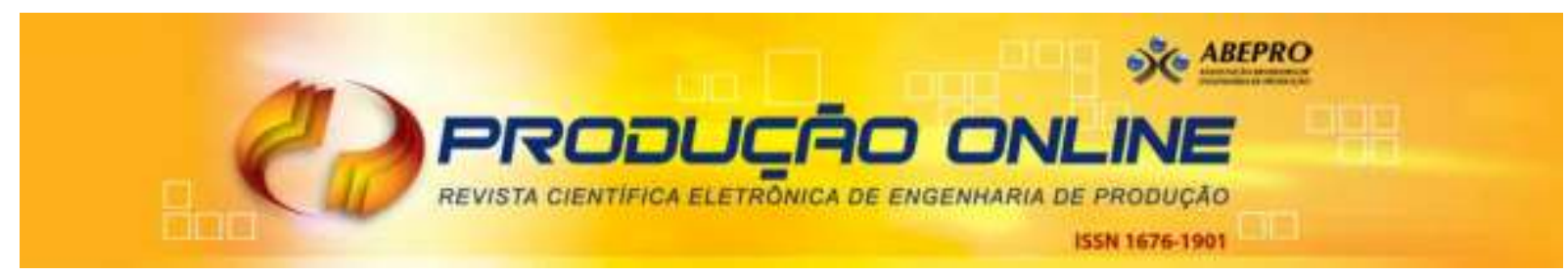

\title{
OTIMIZAÇÃO VIA SIMULAÇÃO POR METAMODELAGEM: UM ESTUDO EM CASOS DA ÁREA MÉDICA
}

\section{SIMULATION OPTIMIZATION USING METAMODELING: A STUDY IN MEDICAL CASES}

\author{
Rafael de Carvalho Miranda* E-mail: rafael.miranda@unifei.edu.br \\ José Arnaldo Barra Montevechi E-mail: montevechi@unifei.edu.br \\ Tábata Fernandes Pereira* E-mail: tabatafp@gmail.com \\ Aneirson Francisco da Silva** E-mail: aneirson@feg.unesp.br \\ *Universidade Federal de Itajubá (UNIFEI), Itajubá, MG, Brasil \\ **Universidade Estadual Paulista (UNESP), Guaratinguetá, SP, Brasil
}

\begin{abstract}
Resumo: O uso combinado da simulação a eventos discretos e otimização vem crescendo nos últimos anos. No entanto, esta forma de otimização ainda é pouco utilizada, devido principalmente a seu elevado custo computacional. Nesse sentido, vários trabalhos visam desenvolver novos métodos de otimização, buscando acelerar este procedimento. Este trabalho faz uso da técnica de metamodelagem, para criar um modelo matemático, que represente uma determinada saída de um modelo de simulação, e proceder com sua otimização sem a necessidade da utilização do modelo simulado. Para exemplificar o método empregado foram utilizados modelos de simulação ligados à área médica. O uso da metamodelagem permitiu otimizar os modelos de simulação, alcançando soluções estatisticamente iguais as encontradas por um otimizador comercial, com a vantagem de reduzir o tempo computacional do processo em mais de $68 \%$ em média. Desta forma, a metamodelagem se apresenta como uma potencial técnica de otimização para a simulação, ao prover soluções de elevada qualidade em um tempo computacional reduzido, quando comparado a métodos de otimização via simulação tradicionais.
\end{abstract}

Palavras-chaves: Otimização via Simulação. Simulação a Eventos Discretos. Metamodelagem. Otimização. Área Médica.

Abstract: The combination of discrete event simulation and optimization techniques is growing in recent years. However, such a combination isn't widely used due mainly to high computational costs. Due to this difficulty, some studies are focused on developing more computationally efficient optimization methods. This article uses a metamodeling technique to create a mathematical model for an output of interest, by running a limited number of scenarios of a simulation model and then proceed to optimization of this output model. We argue that this approach results in significantly reduced computational burden compared to optimizing directly on scenario-specific output values obtained from the simulation model. We illustrate this method using simulation models of distinct functional areas across hospitals in Brazil. The use of metamodeling allows optimization of the simulation models, reaching better solutions than those obtained by a commercial optimizer, with the additional advantage of reducing the computational time by more than $68 \%$ on average.

Keywords: Simulation Optimization. Discrete Events Simulation. Metamodeling. Optimization. Medical Cases. 


\section{INTRODUÇÃO}

A maioria dos problemas associados à otimização de sistemas reais são complexos demais para serem modelados analiticamente. Tal fato decorre da alta variabilidade, da não linearidade dessa classe de problemas e da possibilidade de ocorrência de múltiplos objetivos. Nesse sentido, a Simulação a Eventos Discretos (SED) tem sido uma ferramenta útil para análise de desempenho desses sistemas (LEE et al., 2008).

De fato, a SED vem sendo utilizada de forma crescente a mais de 40 anos, para apoiar a tomada de decisões (BANKS et al., 2009; SARGENT, 2013; LAW, 2007; SIEBERS et al. 2010). Isso se deve, principalmente, a sua versatilidade, flexibilidade e poder de análise, o que lhe permite trabalhar com sistemas altamente complexos (JAHANGIRIAN et al., 2010; RYAN e HEAVEY, 2006).

Para Shen e Wan (2009) e Sharda e Bury (2011), a SED já é apontada como uma das técnicas de Pesquisa Operacional mais utilizadas, permitindo o estudo de diversos sistemas de forma mais econômica, rápida e flexível que a experimentação direta em sistemas reais, como pode ser visto em Pergher et al. (2014), Oliveira, Corrêa e Patrocínio (2014) e Baptista e Rangel (2013).

Apesar de sua crescente utilização, a SED apresenta desvantagens, sendo que uma delas é que ela fornece apenas estimativas estatísticas e não resultados exatos, gerando uma alternativa ideal para um dado problema, apenas se algum método de otimização for utilizado acoplado ao módulo de simulação (STEPONAVIČÉ, RUUSKA e MIETTINEN, 2014; HILLIER e LIEBERMAN, 2010).

Sem o uso conjunto de uma ferramenta de otimização, um analista teria de simular várias configurações do sistema real em busca daquela que apresente o meIhor desempenho, o que muitas vezes é insuficiente do ponto de vista da otimização (LAW e MCCOMAS, 2002; LEE et al., 2008).

De modo a contornar esta limitação, surge o conceito da Otimização via Simulação (OvS) (FU et al., 2000), sendo utilizada de forma crescente a partir da década de 90 , devido principalmente a pacotes de simulação que já possuem rotinas de otimização integradas (FU, 2002; BANKS et al., 2009).

Apesar dos grandes avanços ocorridos, uma crítica comum aos softwares de OvS está relacionada ao custo computacional para se chegar a uma solução. Para vários autores, a grande limitação da OvS está no número de variáveis a serem otimizadas, sendo o desempenho da otimização reduzido drasticamente, em modelos de simulação com muitas variáveis de decisão (MIRANDA et al., 2014; STEPONAVIČĖ, RUUSKA e MIETTINEN, 2014).

Neste cenário, para Villarreal-Marroquín et al. (2013), a motivação para a utilização de técnicas baseadas em metamodelagem para OvS se dá, justamente, pelo elevado custo computacional, necessário para avaliação de uma única execução de um modelo de simulação, que pode variar de alguns minutos até várias horas ou até mesmo dias. 
Para Barton e Meckesheimer (2006), o metamodelo de simulação é um modelo matemático mais simples, que se aproxima do modelo de simulação. Nesse sentido, diferentes técnicas de metamodelagem têm sido utilizadas em OvS, como a Metodologia de Superfície de Resposta (RSM), Metamodelos de Kriging e Redes Neurais Artificiais (RNAs) (VILLARREAL-MARROQUÍN et al., 2013).

A metamodelagem permite a obtenção de um modelo matemático que representa o modelo de simulação, permitindo assim, a otimização deste modelo matemático, de forma mais rápida, que a utilização de um otimizador em conjunto a um simulador a eventos discretos.

Dessa forma, o objetivo deste trabalho é comparar a otimização de modelos de simulação a eventos discretos utilizando a metamodelagem e um otimizador comercial tradicional. Comparando a resposta encontrada em ambas as situações e o tempo envolvido nas otimizações. Os modelos de simulação utilizados para comparação representam casos reais referentes à área médica. O primeiro objeto de estudo utilizado representa uma unidade de emergência hospitalar e foi construído com base no trabalho de Ahmed e Alkhamis (2009), já o segundo objeto de estudo representa uma unidade de processamento de roupas de um hospital brasileiro, o modelo de simulação construído foi baseado no trabalho de Miranda et al. (2012).

Para cumprir com os objetivos, o trabalho está dividido em cinco seções. A segunda seção apresenta a fundamentação teórica do trabalho. A seção três apresenta o método de pesquisa empregado. A seção quatro descreve os objetos de estudos, os passos necessários para obtenção dos metamodelos e suas otimizações. Por fim, a seção cinco apresenta as conclusões do trabalho, seguida dos agradecimentos e das referências bibliográficas.

\section{FUNDAMENTAÇÃO TEÓRICA}

\subsection{Otimização via simulação}

Segundo Swisher et al. (2000), Fu (2002), Ólafsson e Kim (2002) e Eskandari et al. (2011), pode-se definir a Otimização via Simulação como o processo de procurar o melhor conjunto de soluções para um modelo de simulação, ou seja, seus parâmetros ou variáveis de entrada, de forma a otimizar as saídas do modelo.

Um modelo de simulação é composto por $n$ variáveis de entrada $\left(x_{1}, x_{2}, \ldots, x_{n}\right)$ e outras $m$ variáveis de saída $\left(y_{1}, y_{2}, \ldots, y_{m}\right)$ (Figura 1). $O$ uso da otimização visa responder quais os ajustes ótimos das variáveis de entrada $\left(x_{1}, x_{2}, \ldots, x_{n}\right)$ que maximizam (ou minimizam) uma ou mais variáveis de saída $\left(y_{1}, y_{2}, \ldots, y_{m}\right)$ no modelo de simulação (CARSON e MARIA, 1997; HARREL et al., 2002). 
Figura 1 - Modelo de simulação

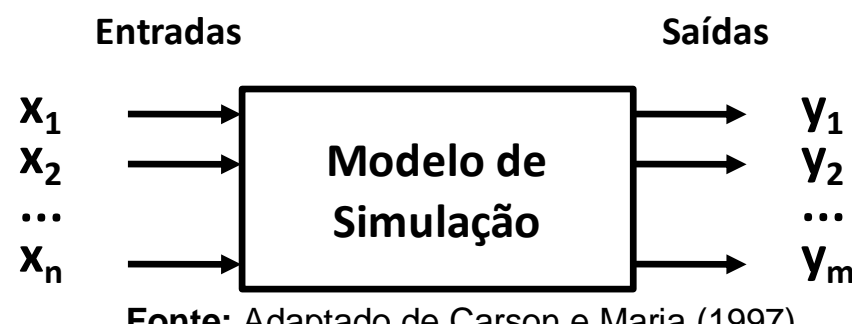

Fonte: Adaptado de Carson e Maria (1997).

Chwif e Medina (2010) destacam como vantagem da OvS, o fato de se ter um procedimento sistemático para a otimização de variáveis, evitando o método de tentativa e erro. Ainda para os autores, com a integração alcançada pela OvS, a simulação passa a ser uma geradora de soluções e não apenas uma avaliadora de possíveis soluções para o problema.

Para Swisher et al. (2000), os parâmetros de entrada de um modelo de simulação podem ser tanto discretos como contínuos, ou até mesmo uma mistura dos dois. Sendo que os tipos de parâmetros de entradas, bem como suas faixas de variação, influenciarão na região de busca e no método de otimização a ser empregado.

Fu (1994) define um problema de OvS, com um único objetivo, como sendo (Eq. 1):

$$
\begin{gathered}
\min f(\theta) \\
\text { Sujeito a: } \theta \in \Theta
\end{gathered}
$$

sendo $f(\theta)=E[\psi(\theta, \omega)]$ o valor esperado do desempenho do sistema, estimado a partir de amostras do modelo de simulação; e $\psi_{j}(\theta, \omega)$ os valores de desempenho observados segundo parâmetros de entrada discretos ou contínuos, pertencentes a um conjunto de soluções viáveis $\Theta$.

Apesar da literatura dar maior destaque à OvS com um único objetivo (WILLIS e JONES, 2008), Jones et al. (2002) comentam, que usualmente os problemas do mundo real possuem múltiplos objetivos, que em geral são conflitantes entre si. Estes autores destacam ainda que, além da complexidade de resolução natural associada à otimização multiobjetivo, em um problema real, deve-se levar em consideração uma possível aleatoriedade nos dados de entrada, o que torna esta tarefa difícil de ser realizada.

Kleijnen, Beers e Nieuwenhuyse (2010) reconhecem os problemas de OvS como sendo, em geral, problemas de difícil solução ou NP-Hard. Estes autores apresentam como desvantagens de sua utilização, o fato das saídas dos modelos de SED serem provenientes de funções implícitas e expostas a ruídos, bem como, dependendo do número de variáveis de entradas no modelo de simulação e de seus intervalos de variação, o processo de otimização pode se tornar complexo, devido às exigências computacionais e ao tempo envolvido para convergência.

Para Fu (2002), o funcionamento de um procedimento de OvS que opera em paralelo a um software de SED pode ser dividido em duas etapas. A primeira consis-

Revista Produção Online, Florianópolis, SC, v. 16, n. 3, p. 1058-1078, jul./set. 2016. 
te em gerar as possíveis candidatas a solução para o problema. Já a segunda consiste em avaliar as soluções, estimando seus resultados. A Figura 2 exemplifica a visão de Fu (2002) sobre o funcionamento da OvS.

Segundo o autor, a OvS concentra-se na primeira etapa, tratando o modelo de simulação como apenas um gerador de funções. Nesse sentido, a maior parte dos cálculos realizados é despendida na estimativa de $f(\theta)$ para cada valor de $\theta$. Sendo um dos principais determinantes do tempo computacional da OvS, o número de replicações necessárias para estimar $f(\theta)$.

Figura 2 - Funcionamento da OvS

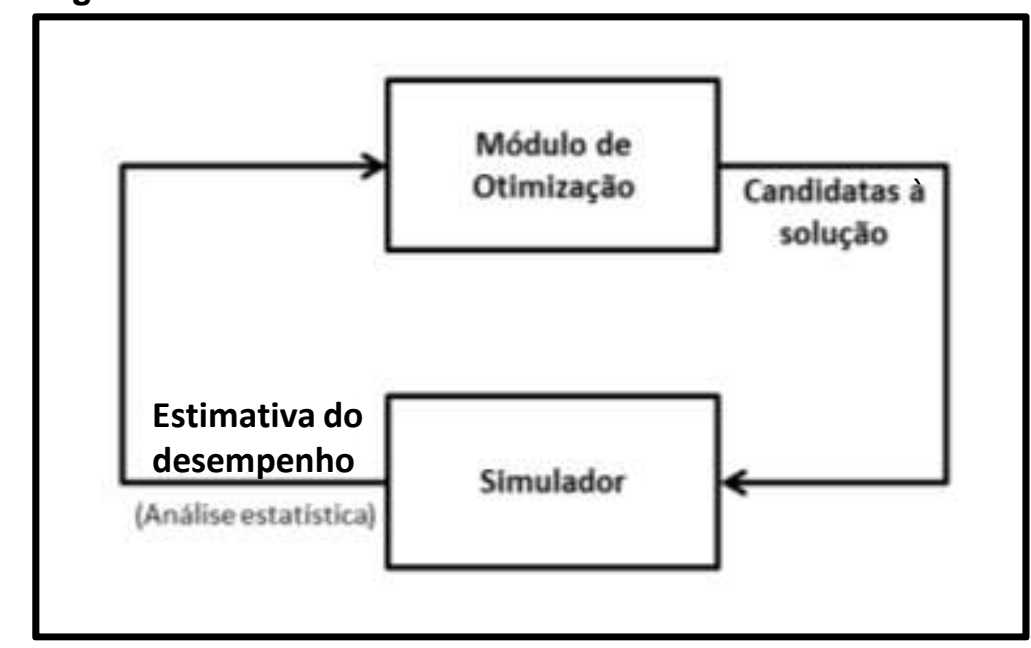

Fonte: Adaptado de Fu (2002)

A abordagem genérica apresentada por Fu (2002) é expandida por Law (2007), para um módulo de OvS baseado em algoritmos evolutivos e algoritmos genéticos (Figura 3). Para Chwif e Medina (2010), esses métodos de otimização são os mais utilizados nos softwares comerciais.

Figura 3 - Princípio de funcionamento de um modulo de otimização integrado à SED

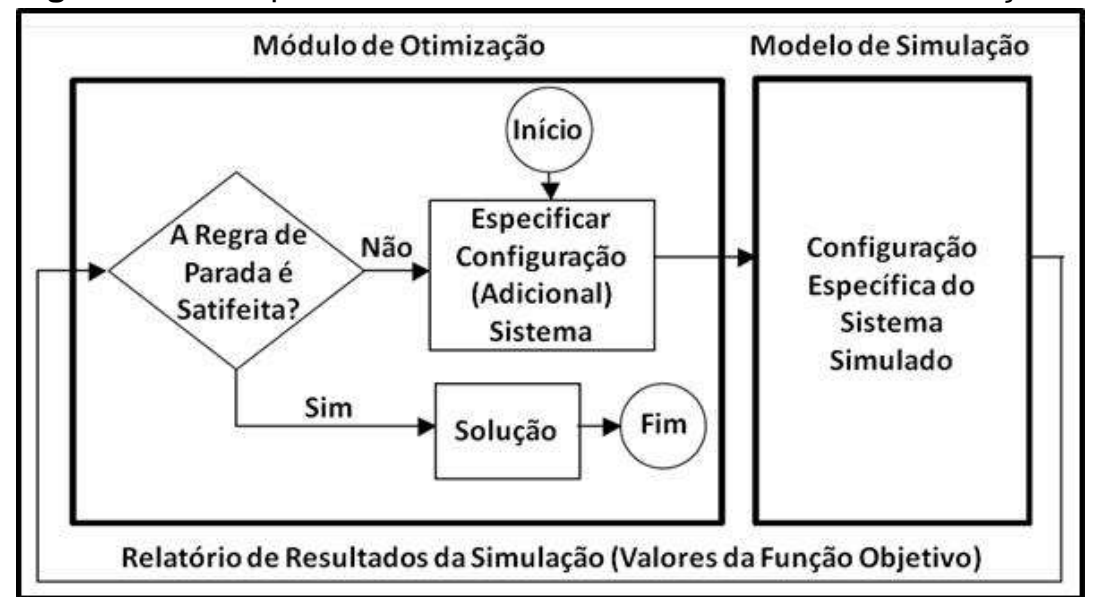

Fonte: Adaptado de Law (2007).

Segundo Law (2007), o módulo de otimização do software envia ao modelo Revista Produção Online, Florianópolis, SC, v. 16, n. 3, p. 1058-1078, jul./set. 2016. 
de simulação as configurações iniciais do sistema (valores iniciais para as variáveis de decisão). O modelo de simulação então, executa as condições especificadas pelo módulo de otimização, avaliando e armazenando a(s) estatística(s) da(s) variável(s) de saída(s) que se pretende otimizar.

Estes resultados são repassados para o módulo de otimização, que dessa forma, usa seu algoritmo de busca, para gerar configurações adicionais para o problema de OvS. Todo o processo é repetido, enquanto todas as restrições do sistema são satisfeitas, até que o critério de parada do módulo de otimização seja alcançado.

Para Wang e Schonfeld (2006), não existem garantias, na maioria dos casos, que os resultados encontrados com um procedimento de OvS sejam ótimos. Estes resultados dependem do método de otimização empregado e de suas configurações. Um bom software de OvS pode alcançar soluções próximas à ideal, ainda que, para estes autores, a diferença entre o ótimo global e a solução apresentada pelo software é normalmente insignificante, considerando a otimização de um modelo de simulação estocástico.

\subsection{Metamodelagem}

Segundo Bettonvil, Castillo e Kleijnen (2009) existem na literatura diversos métodos disponíveis para resolução de problemas como os apresentados na Eq. (1), porém, infelizmente, alguns desses métodos não garantem que a solução encontrada seja uma solução ótima.

Para Rosen, Harmonosky e Traband (2007), o método de otimização aplicável para o problema de OvS dependerá do sistema simulado, se este é composto por variáveis contínuas, discretas ou por ambas.

Kuo, Yang e Huang (2008) destacam o uso da metamodelagem como forma de se otimizar um modelo de SED. Para Cechin e Stertz (2003), metamodelos são aproximações dos resultados de uma simulação, que transformam parâmetros de entrada em parâmetros de saída. Os metamodelos são usados para obter uma visão geral dos resultados, filtrando os resultados da simulação e reduzindo-os a valores médios e aos efeitos principais.

Para Shan e Wang (2010), nas últimas duas décadas, a metamodelagem tem sido desenvolvida para lidar com funções black-box, que possuem alto custo computacional. Segundo Qiu et al. (2016), as técnicas de metamodelagem tem ganhado rápido domínio em problemas complexos na engenharia. Isto pode ser observado por meio da eficiência da modelagem e acuracidade, que estão diretamente associadas com o espaço de amostragem da experimentação, reduzindo a complexidade do espaço e melhorando a acuracidade da modelagem.

A otimização de modelos de simulação por metamodelagem tem sido utilizada recentemente em alguns casos práticos, especialmente onde o tempo e custo para chegar a uma solução viável ou ideal é realmente importante, alguns exemplos podem ser vistos em: Li et al. (2010), Horng e Lin (2013), Fienen, Nolan e Feinstein, 
(2016), Qiu et al. (2016), Hajikolaei, Cheng e Wang (2016), Zeinali, Mahootchi e Sepehri (2015).

Para Jerz e Kralik (2014), os metamodelos podem ser usados para determinação da influência de vários parâmetros de entrada nos resultados, podendo ser criados a partir da avaliação de experimentos em modelos de simulação. Estes podem ser formulados na forma de equações ou em forma de gráficos. Os metamodelos podem ser considerados modelos preditivos descritivos, uma vez que refletem as características do sistema modelado.

\subsubsection{Eureqa Formulize}

Segundo informações da Nutonian (2016), o Eureqa Formulize® possui aplicações em 13 diferentes áreas, que podem ser comprovados por publicações de artigos, algumas dessas áreas são: engenharia, finanças, astronomia, biologia, química, ciência da computação, entre outras.

$\mathrm{Na}$ literatura podem ser encontrados alguns estudos que utilizam o Eureqa Formulize ${ }^{\circledR}$ para conduzir estudos de metamodelagem, tais como: Sakti et al. (2015), Sagdeev et al. (2014) e Akhmedova-Azizova e Abdulagatov (2014).

Neste trabalho, este software será utilizado para geração do metamodelo, que irá representar uma determinada saída de um modelo de simulação, visando sua otimização.

O Eureqa Formulize® (NUTONIAN, 2016) é um software de mineração de dados científicos, que procura por padrões matemáticos em um determinado conjunto de dados (SREČEC et al., 2013). Segundo O'Reilly (2014), o software possui as seguintes características:

- É um programa criado para pesquisar as relações entre diferentes conjuntos de dados;

- Utiliza programação genética para testar diversas funções em um conjunto de dados;

- Cria funções complexas, reduzindo os erros de predição do modelo;

- Inclui uma interface gráfica de fácil utilização para orientar os usuários, por meio da configuração, execução e análise.

Para Gaucel et al. (2014) existe uma característica particular neste software, em vez de retornar uma única solução por execução, ele apresenta ao usuário um conjunto de soluções que representam uma fronteira de Pareto para os objetivos.

\section{MÉTODO DE PESQUISA}

Quanto à natureza, este trabalho pode ser classificado como aplicado, uma vez que, segundo Appolinário (2006), ele possui objetivos práticos, ou seja, que os resultados alcançados com sua realização sejam aplicados ou utilizados para a solução de problemas reais. 
Quanto à abordagem, pode-se classificar este trabalho como uma pesquisa baseada em modelagem quantitativa. Esta classificação é atribuída às pesquisas, nas quais modelos de relações causais entre variáveis de controle e variáveis de desempenho são desenvolvidos, analisados ou testados e se alteram sobre um domínio específico (BERTRAND e FRANSOO, 2002).

Quanto aos objetivos, este trabalho pode ser classificado como uma pesquisa empírica, já que a principal preocupação é assegurar que existe uma adequação entre o modelo desenvolvido e o sistema real. Neste tipo de pesquisa, os resultados teóricos são aplicados em processos reais.

Ainda segundo Bertrand e Fransoo (2002), em uma classificação mais ampla, este trabalho pode ser classificado como uma pesquisa empírica normativa. Uma vez que, neste trabalho, busca-se melhorar uma situação real, ao otimizar modelos que representam problemas de sistemas reais.

Por fim, optou-se pela utilização da modelagem matemática como método de pesquisa para condução do trabalho a ser desenvolvido. Chung (2004) define modelagem como o processo de criar e experimentar um sistema físico, por meio de um modelo matemático. Para Bertrand e Fransoo (2002), este método deve ser usado quando se deseja prever o efeito de mudanças no sistema ou avaliar seu desempenho ou comportamento, sendo utilizado na resolução de problemas reais.

\section{METAMODELAGEM}

Na sequência são apresentadas as fases propostas por Kuo, Yang e Huang (2008) para obtenção do metamodelo e sua otimização.

Para os autores, a otimização de um modelo de SED, por metamodelo, ocorre em três etapas. Primeiro, o modelo que se pretende otimizar é utilizado para gerar um conjunto de dados que contenha a variável de saída que se pretende otimizar. Posteriormente, com auxílio de técnicas de metamodelagem, como regressão ou redes neurais, o metamodelo é gerado. Por fim, este metamodelo é otimizado.

Dois objetos de estudo, ligados à área médica, serão empregados para exemplificar a utilização da metamodelagem na otimização de modelos de simulação a eventos discretos.

\subsubsection{Primeiro objeto de estudo}

O primeiro objeto de estudo utilizado neste trabalho representa uma unidade de emergência hospitalar, e foi construído com base no trabalho de Ahmed e Alkhamis (2009). O processo de atendimento do hospital se inicia quando um paciente chega através das portas do setor de emergência, e termina quando o paciente é liberado ou encaminhado para tratamento futuro.

O paciente passa pela recepcionista que coleta as informações pessoais do paciente. Depois disso, o paciente aguarda a disponibilidade de uma vaga na sala de exame. Nessa sala, o paciente é avaliado por um médico, que irá decidir se o Revista Produção Online, Florianópolis, SC, v. 16, n. 3, p. 1058-1078, jul./set. 2016. 
paciente precisa de mais exames. Então os pacientes são classificados como críticos (categoria 1) ou não-críticos (categorias 2 e 3), de acordo com as suas condições.

Na categoria 2, os pacientes esperam por um tratamento mais simples, que é realizado por uma enfermeira na sala de tratamento. Na categoria 3, os pacientes recebem sua medicação e são liberados do hospital. Cada paciente crítico é encaminhado a um leito na sala de emergência, na qual recebe tratamento. $O$ tratamento na sala de emergência é realizado por uma enfermeira e um médico. Os pacientes que chegam ao hospital em uma ambulância são considerados pacientes críticos (categoria 1), e são encaminhados imediatamente para a sala de emergência. Maiores informações sobre o sistema simulado podem ser obtidas em Ahmed e Alkhamis (2009).

Este objeto de estudo apresenta cinco variáveis de decisão, apresentadas na Tabela 1. Para esse objeto de estudo buscou-se encontrar a melhor combinação das variáveis de entrada, de modo a otimizar a taxa de atendimento horária de pacientes no hospital $\left(y_{1}\right)$.

Tabela 1 - Variáveis de decisão, tipo e limites para o primeiro objeto de estudo

\begin{tabular}{lllcc}
\hline & \multicolumn{1}{c}{ Variável de Decisão } & Tipo & Limite Inferior & Limite Superior \\
\hline$x_{1}$ & Número de recepcionistas & Inteiro & 1 & 3 \\
$x_{2}$ & Número de médicos & Inteiro & 1 & 4 \\
$x_{3}$ & Número de técnicos de laboratório & Inteiro & 1 & 5 \\
$x_{4}$ & Número de enfermeiras na sala de tratamento & Inteiro & 1 & 6 \\
$x_{5}$ & Numero de enfermeiras na sala de emergência & Inteiro & 1 & 12 \\
\hline
\end{tabular}

Considerando o número de variáveis de decisão e o número de níveis por variável, existe um total de $4.320(3 \times 4 \times 5 \times 6 \times 12)$ cenários para a busca da melhor solução.

\subsubsection{Geração do conjunto de dados}

A primeira etapa para o desenvolvimento do metamodelo que irá representar a variável de saída (taxa de atendimento horária de pacientes no hospital) que será otimizada, consiste na geração de um conjunto de dados que relacione as variáveis de entrada com a variável de saída.

Para o presente trabalho optou-se por um Arranjo Ortogonal de Taguchi, como forma de representação do espaço de busca do problema que se deseja otimizar.

Taguchi, Chowdhury e Wu (2005) definem arranjo ortogonal como uma matriz de números dispostos em linhas e colunas, de tal modo que cada par de colunas é ortogonal ao outro. Quando utilizado em um experimento, cada linha representa uma situação experimental, e cada coluna representa um fator específico ou uma determinada condição que pode ser alterada. Para os autores, a matriz é chamada ortogonal, porque os efeitos dos vários fatores podem ser separados uns dos outros.

Revista Produção Online, Florianópolis, SC, v. 16, n. 3, p. 1058-1078, jul./set. 2016. 
A vantagem da utilização dos arranjos ortogonais consiste na capacidade de se avaliar vários fatores com um número mínimo de experimentações. De fato, este arranjo permite considerar $k$ fatores com $n$ níveis cada, testando todos os níveis de cada fator de uma maneira balanceada (ROSS, 1996).

Devido à amplitude de variação dos níveis das variáveis de decisão envolvidas (Tabela 1), optou-se por um arranjo L72. Seguindo a mesma orientação do artigo de Ahmed e Alkhamis (2009), cem replicações, referentes a um dia de operação do hospital, foram realizadas para cada configuração do arranjo L72.

Este arranjo e os resultados simulados por cenário são apresentados na Tabela 2. O resultado referente à variável de saída $\left(y_{1}\right)$ refere-se à média de cem replicações de um dia de operação do hospital.

As simulações dos 72 cenários e suas replicações foram realizadas em um computador com processador Intel (Core 2 Duo) $1.58 \mathrm{GHZ}, 2 \mathrm{~GB}$ de RAM e sistema operacional Windows, plataforma 64 bits e consumiram pouco menos de 8,5 horas.

Tabela 2 - Arranjo ortogonal e resultados obtidos para o primeiro objeto de estudo

\begin{tabular}{|c|c|c|c|c|c|c|c|c|c|c|c|c|c|}
\hline \multirow[t]{2}{*}{ Cenário } & \multicolumn{5}{|c|}{$\begin{array}{l}\text { Variáveis de } \\
\text { Decisão }\end{array}$} & \multirow{2}{*}{$\begin{array}{c}\text { Variáveis de Saída } \\
y_{1}\end{array}$} & \multirow[t]{2}{*}{ Cenário } & \multicolumn{5}{|c|}{$\begin{array}{l}\text { Variáveis de } \\
\text { Decisão }\end{array}$} & \multirow{2}{*}{$\begin{array}{c}\begin{array}{l}\text { Variáveis } \\
\text { de Saída }\end{array} \\
y_{1}\end{array}$} \\
\hline & $x_{1}$ & $x_{2}$ & $x_{3}$ & $x_{4}$ & $y_{1}$ & & & $x_{1}$ & $x_{2}$ & $x_{3}$ & $x_{4}$ & $x_{5}$ & \\
\hline 1 & 3 & 1 & 4 & 4 & 1 & 1,71 & 37 & 2 & 1 & 1 & 3 & 7 & 2,65 \\
\hline 2 & 3 & 2 & 5 & 3 & 1 & 2,22 & 38 & 3 & 2 & 5 & 4 & 7 & 5,10 \\
\hline 3 & 2 & 4 & 2 & 6 & 1 & 2,41 & 39 & 1 & 3 & 4 & 6 & 7 & 5,72 \\
\hline 4 & 2 & 1 & 2 & 5 & 1 & 1,68 & 40 & 3 & 3 & 3 & 1 & 7 & 5,58 \\
\hline 5 & 1 & 2 & 1 & 2 & 1 & 2,07 & 41 & 2 & 4 & 5 & 5 & 7 & 5,74 \\
\hline 6 & 1 & 3 & 3 & 1 & 1 & 2,35 & 42 & 1 & 2 & 2 & 2 & 7 & 4,71 \\
\hline 7 & 2 & 4 & 4 & 2 & 2 & 4,16 & 43 & 3 & 2 & 2 & 5 & 8 & 4,80 \\
\hline 8 & 3 & 1 & 2 & 1 & 2 & 2,47 & 44 & 3 & 2 & 3 & 6 & 8 & 5,13 \\
\hline 9 & 1 & 3 & 5 & 6 & 2 & 3,98 & 45 & 2 & 3 & 4 & 1 & 8 & 5,61 \\
\hline 10 & 2 & 4 & 1 & 3 & 2 & 2,96 & 46 & 2 & 4 & 3 & 3 & 8 & 5,73 \\
\hline 11 & 1 & 2 & 3 & 4 & 2 & 3,64 & 47 & 1 & 1 & 1 & 4 & 8 & 2,63 \\
\hline 12 & 3 & 1 & 2 & 5 & 2 & 2,44 & 48 & 1 & 1 & 5 & 2 & 8 & 2,70 \\
\hline 13 & 1 & 4 & 3 & 5 & 3 & 5,26 & 49 & 2 & 3 & 5 & 3 & 9 & 5,75 \\
\hline 14 & 2 & 2 & 5 & 4 & 3 & 4,47 & 50 & 3 & 2 & 4 & 6 & 9 & 5,06 \\
\hline 15 & 1 & 1 & 5 & 1 & 3 & 2,66 & 51 & 3 & 1 & 4 & 5 & 9 & 2,79 \\
\hline 16 & 3 & 4 & 1 & 2 & 3 & 2,99 & 52 & 1 & 2 & 2 & 2 & 9 & 4,72 \\
\hline 17 & 2 & 1 & 2 & 6 & 3 & 2,68 & 53 & 2 & 3 & 1 & 4 & 9 & 2,96 \\
\hline 18 & 3 & 3 & 4 & 3 & 3 & 5,11 & 54 & 1 & 4 & 3 & 1 & 9 & 5,59 \\
\hline 19 & 3 & 3 & 1 & 6 & 4 & 3,01 & 55 & 3 & 4 & 2 & 4 & 10 & 5,07 \\
\hline 20 & 2 & 1 & 3 & 2 & 4 & 2,76 & 56 & 1 & 1 & 3 & 6 & 10 & 2,70 \\
\hline 21 & 3 & 4 & 5 & 5 & 4 & 5,71 & 57 & 3 & 2 & 3 & 3 & 10 & 5,15 \\
\hline 22 & 2 & 2 & 4 & 1 & 4 & 4,75 & 58 & 2 & 2 & 4 & 5 & 10 & 5,06 \\
\hline 23 & 1 & 3 & 2 & 3 & 4 & 4,96 & 59 & 1 & 4 & 1 & 1 & 10 & 3,02 \\
\hline 24 & 1 & 4 & 2 & 4 & 4 & 5,00 & 60 & 2 & 3 & 5 & 2 & 10 & 5,75 \\
\hline 25 & 1 & 2 & 1 & 5 & 5 & 2,99 & 61 & 2 & 2 & 1 & 1 & 11 & 3,03 \\
\hline 26 & 1 & 2 & 4 & 3 & 5 & 4,96 & 62 & 3 & 3 & 2 & 2 & 11 & 5,10 \\
\hline
\end{tabular}


Tabela 2 - Arranjo ortogonal e resultados obtidos para o primeiro objeto de estudo

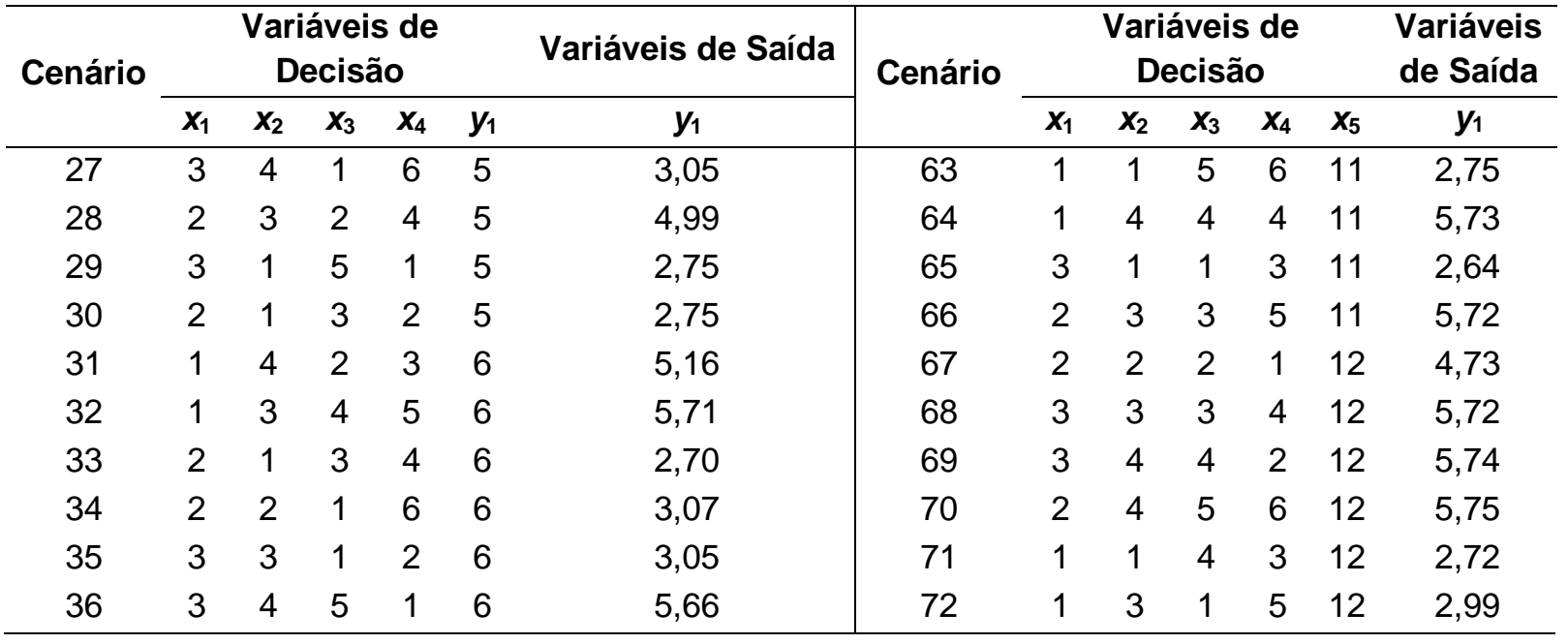

\subsubsection{Construção do metamodelo}

De posse dos dados simulados, o Eureqa Formulize $\AA^{\circledR}$ foi configurado para geração do metamodelo. Nesta etapa utilizou-se o conjunto básico de funções (soma, subtração, multiplicação, divisão e negação) e símbolos terminais (constante inteira, constante flutuante e as variáveis) do software. No computador utilizado para os experimentos, a geração do metamodelo levou em torno de 35 minutos.

O modelo matemático gerado pelo software para a variável de saída: taxa de atendimento horária de pacientes no hospital $\left(y_{1}\right)$, é apresentado na Eq. (2):

$$
\begin{gathered}
y_{1}=1,113 x_{2}+0,701 x_{2} x_{3}+0,207 x_{2} x_{5}+0,021 x_{1} x_{5}-0,435 x_{2}^{2}-0,013 x_{2} x_{5}^{2}- \\
0,092 x_{2} x_{3}^{2}
\end{gathered}
$$

Pode ser observado no modelo matemático (Eq. 2), que a variável $x_{4}$ (número de enfermeiras na sala de tratamento) não foi significativa para a construção do metamodelo. Ficando esta variável no seu nível mínimo $\left(x_{4}=1\right)$ no processo de otimização.

O modelo gerado pelo software apresentou $R^{2}$-Adj de $89,40 \%$, um erro máximo de 1,51 e um erro médio absoluto de 0,22.

\subsubsection{Otimização}

A etapa de otimização foi conduzida em duas etapas para fins de comparação. Primeiro, o metamodelo foi otimizado utilizando o algoritmo Gradiente Reduzido Generalizado (GRG) com auxílio do Solver do Exceß®. Posteriormente, o otimizador SimRunner® foi utilizado para otimização do modelo de simulação.

Para otimização considerou-se como restrições, o limite de variação para cada variável de decisão apresentado na Tabela 1 , e o fato que as variáveis de decisão deveriam ser inteiras. Os resultados encontrados com as otimizações são 
apresentados na Tabela 3.

A resposta encontrada para $y_{1}$, quando adotados os valores encontrados pelo Solver $\left(x_{1}=3, x_{2}=4, x_{3}=4, x_{4}=1, x_{5}=9\right)$ leva a uma resposta igual a 6,63 pacientes/hora atendidos. No entanto, substituindo estes valores no modelo de simulação e realizando as 100 replicações, chega-se a um valor de 5,76 pacientes/hora, valor este apresentado na Tabela 3.

Tabela 3 - Resultados da otimização Metamodelo x SimRunner® para o primeiro objeto de estudo

\begin{tabular}{ccc}
\hline \multirow{2}{*}{ Variáveis de Decisão } & \multicolumn{2}{c}{ Solução } \\
\cline { 2 - 3 } & Metamodelo SimRunner \\
\hline$x_{1}$ & 3 & 3 \\
$x_{2}$ & 4 & 4 \\
$x_{3}$ & 1 & 5 \\
$x_{4}$ & 9 & 6 \\
$x_{5}$ & \multicolumn{2}{c}{ Respostas } \\
\hline Variáveis de Saída & 5,76 & 5,77 \\
\hline$y_{1}$ & $(5,75-5,78)$ & $(5,76-5,78)$ \\
\hline
\end{tabular}

Analisando as variáveis de entrada apresentadas na Tabela 3, verifica-se que a otimização via metamodelo levou a necessidade de um número igual ou menor de recursos que a solução encontrada pelo SimRunner ${ }^{\circledR}$.

Apesar dos valores serem diferentes para a solução encontrada $\left(y_{1}\right)$, as soluções puderam ser consideradas estatisticamente iguais, por meio do teste não paramétrico de Mann-Whitney, que apresentou $p$-value igual 0,279, maior que o nível de significância adotado de $0,05(\alpha=5 \%)$.

Apesar da igualdade das soluções, os tempos envolvidos no processo de otimização foram consideravelmente distintos. O Solver do Exceß® chegou à solução apresentada na Tabela $3 \mathrm{em}$ menos de 1 segundo. Somando a isso, o tempo necessário para geração do número de dados ( 8,5 horas) e o tempo gasto para a geração do metamodelo (35 minutos), chega-se ao tempo total de 9,08 horas. Já o Simrunner conduzindo a otimização do modelo de simulação levou 25,2 horas para chegar à solução apresentada na Tabela 3 . Com a utilização da metamodelagem ocorreu uma redução de aproximadamente $64 \%$ no tempo envolvido na otimização.

\subsubsection{Segundo objeto de estudo}

O segundo objeto de estudo deste trabalho é uma unidade de processamento de roupas (UPR) de um hospital brasileiro. O modelo foi construído com base no trabalho de Miranda et al. (2012).

A UPR é responsável pelo fornecimento e limpeza das roupas utilizadas por usuários e funcionários do hospital que estão diretamente relacionados com o atendimento dos pacientes. O setor é dividido em duas partes: a área contaminada, que 
recebe as roupas provenientes do hospital, e a área limpa ou não contaminada, que recebe as roupas que passaram por processos iniciais de limpeza.

As operações realizadas na UPR são divididas de acordo com a área em que as roupas se encontram e seu grau de contaminação. Na área contaminada, são realizados os processos de recebimento, separação, pesagem, pré-lavagem e lavagem. Na área não contaminada, realizam-se os processos de centrífuga, secagem, calandragem, dobragem, armazenamento e transporte das roupas novamente para o hospital.

O processo inicia com a chegada das roupas na área contaminada. Estas são recebidas pelo funcionário desta área e separadas de acordo com o grau de sujeira e contaminação. De acordo com o grau de sujeira das roupas elas podem seguir para a pré-lavagem. No processo de pré-lavagem as roupas são lavadas manualmente pelo funcionário e na sequência são enviadas para a máquina de lavar.

Se a pré-lavagem não for necessária, as roupas seguem para a pesagem, na qual são separadas em lotes de $30 \mathrm{~kg}$ (capacidade da máquina) e então lavadas na máquina de lavar. Depois de passarem pela máquina de lavar, as roupas são encaminhadas para a área não contaminada. Todos os processos realizados na área contaminada são conduzidos por um único funcionário.

$\mathrm{Na}$ área não contaminada, as roupas que saem da máquina de lavar antes de serem centrifugadas são separadas em lotes de $15 \mathrm{~kg}$, devido à capacidade do equipamento. Isso é feito por outro funcionário do setor. Na sequência, as roupas são enviadas para a secadora. Da secadora as roupas seguem para a calandra (quando necessário) ou diretamente para a dobragem. Após esse processo as roupas são enviadas para carrinhos transportadores, onde são transportadas para os respectivos setores do hospital.

Outras informações a respeito do modelo de simulação podem ser encontradas em Miranda et al. (2012). Ressalta-se, que todas as etapas de verificação e validação do modelo de simulação foram empregadas, de forma que o modelo representa de forma adequada o sistema ao qual se procura otimizar.

Este objeto de estudo apresenta cinco variáveis de decisão, apresentadas na Tabela 4. O objetivo da otimização foi encontrar a melhor combinação das variáveis de decisão, de forma a maximizar o total de roupas lavadas $\left(y_{1}\right)$, em uma semana na lavanderia.

Tabela 4 - Variáveis de decisão, tipo e limites para o segundo objeto de estudo

\begin{tabular}{|c|c|c|c|c|}
\hline & Variável & Tipo & Limite Inferior & Limite Superior \\
\hline$X_{1}$ & Número de lavadoras & Inteira & 1 & 5 \\
\hline$x_{2}$ & Número de centrífugas & Inteira & 1 & 5 \\
\hline$x_{3}$ & Número de secadoras & Inteira & 1 & 5 \\
\hline$X_{4}$ & Número de operadores área contaminada & Inteira & 1 & 5 \\
\hline$x_{5}$ & Número de operadores área não contaminada & Inteira & 1 & 5 \\
\hline
\end{tabular}


Para o problema em questão, considerando o número de variáveis de decisão e sua variação máxima, existe um total de $3.125\left(5^{5}\right)$ cenários possíveis para a busca da melhor configuração.

\subsubsection{Geração do conjunto de dados}

Considerando a quantidade de variáveis de decisão e a variação dos níveis de cada variável, optou-se por um arranjo ortogonal de Taguchi L25. Este arranjo prevê a realização de 25 experimentos.

Com o arranjo definido, foi gerada a matriz experimental, apresentada na Tabela 5. Os cenários na matriz experimental foram simulados no ProModel $\AA^{\circledR}$. Foram simuladas 30 replicações para cada cenário, referentes a uma semana de operação da unidade de processamento de roupas, e os dados referentes a cada variável de saída foram armazenados para construção do metamodelo.

As simulações dos 25 cenários e suas replicações consumiram pouco menos de cinco minutos. Os resultados encontram-se apresentados na Tabela 5. Os dados analisados referem-se às médias das 30 replicações.

Tabela 5 - Arranjo ortogonal e resultados obtidos para o segundo objeto de estudo

\begin{tabular}{|c|c|c|c|c|c|c|}
\hline \multirow{2}{*}{ Cenário } & \multicolumn{5}{|c|}{ Variáveis de Decisão } & \multirow{2}{*}{$\begin{array}{c}\text { Variável de Saída } \\
y_{1}(\mathrm{Kg})\end{array}$} \\
\hline & $x_{1}$ & $x_{2}$ & $x_{3}$ & $x_{4}$ & $x_{5}$ & \\
\hline 1 & 1 & 1 & 1 & 1 & 1 & 2958,00 \\
\hline 2 & 1 & 2 & 2 & 2 & 2 & 2984,00 \\
\hline 3 & 1 & 3 & 3 & 3 & 3 & 3013,00 \\
\hline 4 & 1 & 4 & 4 & 4 & 4 & 2990,00 \\
\hline 5 & 1 & 5 & 5 & 5 & 5 & 3012,00 \\
\hline 6 & 2 & 1 & 2 & 3 & 4 & 5294,00 \\
\hline 7 & 2 & 2 & 3 & 4 & 5 & 5325,00 \\
\hline 8 & 2 & 3 & 4 & 5 & 1 & 4573,00 \\
\hline 9 & 2 & 4 & 5 & 1 & 2 & 5300,00 \\
\hline 10 & 2 & 5 & 1 & 2 & 3 & 5294,00 \\
\hline 11 & 3 & 1 & 3 & 5 & 2 & 5275,00 \\
\hline 12 & 3 & 2 & 4 & 1 & 3 & 5326,00 \\
\hline 13 & 3 & 3 & 5 & 2 & 4 & 5313,00 \\
\hline 14 & 3 & 4 & 1 & 3 & 5 & 5329,00 \\
\hline 15 & 3 & 5 & 2 & 4 & 1 & 4519,00 \\
\hline 16 & 4 & 1 & 4 & 2 & 5 & 5315,00 \\
\hline
\end{tabular}

(continua) 
Tabela 5 - Arranjo ortogonal e resultados obtidos para o segundo objeto de estudo

\begin{tabular}{ccccccc}
\hline \multirow{2}{*}{ Cenário } & \multicolumn{3}{c}{ Variáveis de Decisão } & Variável de Saída \\
\cline { 2 - 7 } & $\boldsymbol{x}_{\mathbf{1}}$ & $\boldsymbol{x}_{\mathbf{2}}$ & $\boldsymbol{x}_{\mathbf{3}}$ & $\boldsymbol{x}_{\mathbf{4}}$ & $\boldsymbol{x}_{\mathbf{5}}$ & $\boldsymbol{y}_{\mathbf{1}}(\mathbf{K g})$ \\
\hline 17 & 4 & 2 & 5 & 3 & 1 & 4530,00 \\
18 & 4 & 3 & 1 & 4 & 2 & 5286,00 \\
19 & 4 & 4 & 2 & 5 & 3 & 5295,00 \\
20 & 4 & 5 & 3 & 1 & 4 & 5288,00 \\
21 & 5 & 1 & 5 & 4 & 3 & 5249,00 \\
22 & 5 & 2 & 1 & 5 & 4 & 5287,00 \\
23 & 5 & 3 & 2 & 1 & 5 & 5328,00 \\
24 & 5 & 4 & 3 & 2 & 1 & 4535,00 \\
25 & 5 & 5 & 4 & 3 & 2 & 5304,00 \\
\hline
\end{tabular}

(conclusão)

\subsubsection{Construção do metamodelo}

De posse dos dados simulados, o Eureqa Formulize $\AA^{\circledR}$ foi configurado para geração do metamodelo. As mesmas configurações apresentadas para o objeto de estudo anterior foram utilizadas para este objeto de estudo. No computador utilizado para os experimentos, a geração do metamodelo levou em torno de 4 minutos.

O modelo matemático gerado pelo software para a variável de saída: total de roupas lavadas $\left(y_{1}\right)$, é apresentado na Eq. (4):

$$
y_{1}=12418,9-7423,6 x_{1}^{-1}+211,03 x_{1}^{2}-254,43 x_{1} x_{5}^{-1}-2094,88 x_{1}
$$

Pode ser observado no modelo matemático (Eq. 3) que as variáveis $x_{2}, x_{3}$ e $x_{4}$ (número de centrífugas, secadoras e operadores na área contaminada) não foram significativas para a construção do metamodelo. Ficando estas variáveis em seu nível mínimo $\left(x_{2}=x_{3}=x_{4}=1\right)$ no processo de otimização.

O modelo gerado pelo software apresentou $\mathrm{R}^{2}$-Adj de $98,04 \%$, um erro máximo de $279,57 \mathrm{~kg}$ e um erro médio absoluto de 98,07 kg.

\subsubsection{Otimização}

A etapa de otimização foi conduzida em duas etapas para fins de comparação. Primeiro, o metamodelo foi otimizado utilizando o algoritmo Gradiente Reduzido Generalizado (GRG) com auxílio do Solver do Exceß. Posteriormente, o otimizador SimRunner® foi utilizado para otimização do modelo de simulação.

Para otimização considerou-se como restrições o limite de variação para cada variável de decisão apresentado na Tabela 4, e o fato que as variáveis de decisão deveriam ser inteiras. Os resultados encontrados com as otimizações são apresentadas na Tabela 6.

A resposta encontrada para $y_{1}$, quando adotados os valores encontrados pelo Solver $\left(x_{1}=5, x_{2}=1, x_{3}=1, x_{4}=1, x_{5}=5\right)$ leva a uma resposta igual a $5481,10 \mathrm{~kg}$ de 
roupas lavadas em uma semana. No entanto, substituindo estes valores no modelo de simulação e realizando as 30 replicações, chega-se a um valor de $5310,00 \mathrm{~kg}$ de roupas lavadas em uma semana, valor este apresentado na Tabela 6.

Tabela 6 - Resultados da otimização Metamodelo x SimRunner® para o segundo objeto de estudo

\begin{tabular}{ccc}
\hline \multirow{2}{*}{ Variáveis de Decisão } & \multicolumn{2}{c}{ Solução } \\
\cline { 2 - 3 } & Metamodelo & SimRunner \\
\hline$x_{1}$ & 5 & 5 \\
$x_{2}$ & 1 & 4 \\
$x_{3}$ & 1 & 1 \\
$x_{4}$ & 1 & 1 \\
$x_{5}$ & 5 & 5 \\
\hline Variáveis de Saída & \multicolumn{2}{c}{ Respostas } \\
\hline$y_{1}$ & 5310,00 & 5363,00 \\
Intervalo de confiança para $y_{1}(95 \%)$ & $(5264,00-5375,00)$ & $(5319,00-5406,00)$ \\
\hline
\end{tabular}

Analisando as variáveis de decisão apresentadas na Tabela 6 , verifica-se que a otimização via metamodelo levou a necessidade de um número igual ou menor de recursos que a solução encontrada pelo SimRunner.

Apesar dos valores serem diferentes para a solução encontrada $\left(y_{1}\right)$, as soluções puderam ser consideradas estatisticamente iguais, por meio do teste não paramétrico de Mann-Whitney, que apresentou $p$-value igual 0,200, maior que o nível de significância adotado de $0,05(\alpha=5 \%)$.

Apesar da igualdade das soluções, os tempos envolvidos no processo de otimização foram consideravelmente distintos. O Solver do Excel® chegou à solução apresentada na Tabela $6 \mathrm{em}$ menos de 1 segundo. Somando-se a isso, o tempo necessário para geração do número de dados (5 minutos) e o tempo gasto para a geração do metamodelo (4 minutos), chega-se ao tempo total de 9 minutos. Já o Simrunne! ${ }^{\circledR}$ conduzindo a otimização do modelo de simulação levou 33 minutos para chegar à solução apresentada na Tabela 6 , ou seja, com a utilização da metamodelagem ocorreu uma redução no tempo do processo de otimização de mais de $72 \%$.

\section{CONCLUSÕES}

A utilização da otimização em conjunto com a simulação tem avançado de forma crescente nas últimas três décadas, no entanto, vários desafios se impõem a sua utilização. Dentre estes desafios, destaca-se o elevado custo computacional envolvido neste tipo de otimização.

Este trabalho conduziu uma comparação da otimização de modelos de simulação a eventos discretos, utilizando a metamodelagem e um otimizador comercial tradicional. Estes experimentos foram estudados em dois modelos de simulação que representam casos reais referentes à área médica. O primeiro representou uma 
unidade de emergência hospitalar e o segundo representou uma unidade de processamento de roupas de um hospital brasileiro.

Dessa forma, pode-se verificar que a utilização da metamodelagem pode acelerar o processo de otimização via simulação nos objetos de estudos analisados, quando comparado a técnicas tradicionais realizadas por um otimizador comercial, sem que houvesse perda de qualidade da resposta encontrada.

Destaca-se também, a utilização do software Eureqa Formulize®, que permitiu alcançar modelos matemáticos com elevados ajustes e baixas taxas de erros, a partir de cenários gerados pelo modelo de simulação, de forma rápida e sem maiores complexidades de utilização por parte do usuário.

Com o desenvolvimento do metamodelo e sua posterior otimização, houve uma redução de aproximadamente $68 \%$ no tempo envolvido no processo de otimização ao ser comparado com o uso do SimRunner®, alcançando soluções estatisticamente iguais para ambos objetos de estudo. Outro fato a ser destacado foi que a solução alcançada com o metamodelo exigiu um número menor de recursos, em ambos os casos, quando comparada a otimização conduzida pelo SimRunner.

Dessa forma, destaca-se que a metamodelagem pode acelerar o processo de otimização, ao reduzir o tempo necessário para obtenção de uma solução para otimização de um modelo de simulação.

Trabalhos futuros poderão testar outras técnicas de metamodelagem e trabalhar com a otimização de mais de um objetivo, comparando os resultados encontrados com um otimizador comercial.

\section{AGRADECIMENTOS}

Os autores agradecem a CAPES, CNPq, FAPEMIG e FAPESP.

\section{REFERÊNCIAS}

AHMED, M. A.; ALKHAMIS, T. M. Simulation optimization for an emergency department healthcare unit in Kuwait. European Journal of Operational Research, v.198, n.3, p.936942, 2009. https://doi.org/10.1016/j.procs.2012.04.161

AKHMEDOVA-AZIZOVA, L. A.; ABDULAGATOV, I. M. Thermal Conductivity of Aqueous $\mathrm{CaCl} 2$ Solutions at High Temperatures and High Pressures. Journal of Solution Chemistry, v. 43, n. 3, p. 421-444, 2014. https://doi.org/10.1007/s10953-014-0141-z

APPOLINÁRIO, F. Metodologia da ciência: filosofia e prática da pesquisa. São Paulo: Editora Pioneira Thomson Learning, 2006.

BANKS, J.; CARSON II, J. S.; NELSON, B. L.; NICOL, D. M. Discrete-event Simulation. $5^{\text {th }}$. ed., New Jersey: Prentice-Hall, 2009.

BAPTISTA, R. C. T.; RANGEL, J. J. A. Simulação a eventos discretos de uma via semaforiRevista Produção Online, Florianópolis, SC, v. 16, n. 3, p. 1058-1078, jul./set. 2016. 
zada com controle automatizado em tempo real. Revista Produção Online, v. 13, n. 1, p. 290-317, 2013. https://doi.org/10.14488/1676-1901.v13i1.1149

BARTON, R.R.; MECKESHEIMER, M. Metamodel-based simulation optimization. In: BIRGE, J. R.; LINETSKY, V. (Eds.). Handbooks in operations research and management science, Cap.18, p.535-574, 2006.

BERTRAND, J. W. M.; FRANSOO, J. C. Modelling and simulation: operations management research methodologies using quantitative modeling. International Journal of Operations \& Production Management, v.22, n.2, p.241-264, 2002.

https://doi.org/10.1108/01443570210414338

BETTONVIL, B. W. M.; CASTILLO, E.; KLEIJNEN, J. P. C. Statistical testing of optimality conditions in multiresponse simulation-based optimization. European Journal of Operational Research, v.199, n.2, p.448-458, 2009. https://doi.org/10.2139/ssrn.995411

CARSON, Y.; MARIA, A. Simulation optimization: methods and applications. In: Winter Simulation Conference, Proceedings... Atlanta, GA, USA, 1997.

https://doi.org/10.1145/268437.268460

CECHIN, A. L.; STERTZ, K. Comparando a performance de redes neurais artificiais como metamodelos de simulação. In: XXIII Encontro Nacional de Engenharia de Produção,

Anais... Ouro Preto, MG, Brasil, 2003.

CHUNG, C. A. Simulation modeling handbook: a practical approach. Washington, D.C: CRC Press, 2004.

CHWIF, L.; MEDINA, A. C. Modelagem e simulação de eventos discretos: teoria e aplicações. 2. ed. São Paulo: Editora dos Autores, 2010.

ESKANDARI, H.; MAHMOODI, E.; FALLAH, H.; GEIGER, C. D. Performance analysis of comercial simulation-based optimization packages: OptQuest and Witness Optimizer. In: Winter Simulation Conference, Proceedings... Phoenix, AZ, USA, 2011.

https://doi.org/10.1109/WSC.2011.6147946

FIENEN, M. N.; NOLAN, B. T.; FEINSTEIN, D. T. Evaluating the sources of water to wells: Three techniques for metamodeling of a groundwater flow model. Environmental Modelling \& Software, v. 77, p. 95-107, 2016. https://doi.org/10.1016/j.envsoft.2015.11.023

FU, M. C. Optimization via simulation: A review. Annals of Operations Research, v. 53, p.199-247, 1994. https://doi.org/10.1007/BF02136830

FU, M.C. Optimization for Simulation: Theory vs. Practice. Journal on Computing, v.14, n.3, p. 192-215, 2002. https://doi.org/10.1287/ijoc.14.3.192.113

FU, M. C.; ANDRADÓTTIR, S.; CARSON, J. S.; GLOVER, F.; HARRELL, C. R.; HO, Y. C.; KELLY, J. P.; ROBINSON, S. M. Integrating optimization and simulation: research and practice. In: Winter Simulation Conference, Proceedings... Orlando, FL, USA, 2000.

https://doi.org/10.1109/WSC.2000.899770

GAUCEL, S.; KEIJZER, M.; LUTTON, E.; TONDA, A. Learning Dynamical Systems Using Standard Symbolic Regression. In: Genetic Programming. Proceedings... Springer Berlin Heidelberg, 2014. https://doi.org/10.1007/978-3-662-44303-3 3 
HAJIKOLAEI, K. H.; CHENG, G. H.; WANG, G. G. Optimization on Metamodeling-Supported Iterative Decomposition. Journal of Mechanical Design, v. 138, n. 2, p. 021401, 2016. https://doi.org/10.1115/1.4031982

HARREL, C. R.; MOTT, J. R. A; BATEMAN, R. E.; BOWDEN, R. G.; GOGG, T. J. Simulação: otimizando os sistemas. 2.ed. São Paulo: IMAM, 2002.

HILLIER, F. S.; LIEBERMAN, G. J.; Introduction to Operations Research. $9^{\text {th }}$. ed. New York: McGraw-Hill, 2010.

HORNG, S.; LIN, S. Evolutionary algorithm assisted by surrogate model in the framework of ordinal optimization and optimal computing budget allocation. Information Sciences, v.233, p.214-229, 2013. https://doi.org/10.1016/j.ins.2013.01.024

JAHANGIRIAN, M.; ELDABI, T.; NASEER, A.; STERGIOULAS, L. K.; YOUNG, T. Simulation in manufacturing and business: A review. European Journal of Operational Research, v.203, n.1, p.1-13, 2010. https://doi.org/10.1016/j.ejor.2009.06.004

JERZ, V.; KRALIK, M. The use of metamodels to interpret the results of simulation studies. IJMCA, v.2, n.6, p.177-181, 2014.

JONES, D. F.; MIRRAZAVI, S. K.; TAMIZ, M. Multi-objective meta-heuristics: an over-view of the current state-of-the-art. European Journal of Operational Research, v.137, p.1-9, 2002. https://doi.org/10.1016/S0377-2217(01)00123-0

KLEIJNEN, J. P. C.; VAN BEERS, W.; VAN NIEUWENHUYSE, I. Constrained optimization in simulation: A novel approach. European Journal of Operational Research, v.202, n.1, p.164-174, 2010. https://doi.org/10.1016/j.ejor.2009.05.002

KUO, Y.; YANG, T.; HUANG, G. W. The use of a grey-based Taguchi method for optimizing multi-response simulation problems. Engineering Optimization, v.40, n.6, p.517-528, 2008. https://doi.org/10.1080/03052150701857645

LAW, A. M. Simulation modeling and analysis. $4^{\text {th }}$.ed. New York: McGraw-Hill, 2007.

LAW, A. M.; MCCOMAS, M. G. Simulation-Based Optimization, In: Winter Simulation Conference, Proceedings... San Diego, CA, USA, 2002.

LEE, L.H.; CHEW, E. P.; TENG, S.; CHEN, Y. Multi-objective simulation-based evolutionary algorithm for an aircraft spare parts allocation problem. European Journal of Operational Research, v.189, p.476-491, 2008. https://doi.org/10.1016/j.ejor.2007.05.036

LI, Y. F.; NG, S. H.; XIE, M.; GOH, T. N. A systematic comparison of metamodeling techniques for simulation optimization in decision support systems. Applied Soft Computing, v.10, n.4, p.1257-1273, 2010.https://doi.org/10.1016/j.asoc.2009.11.034

MIRANDA, R. C.; MONTEVECHI, J. A. B.; PENTEADO, K. M.; AGUIAR, M. S.; PINHO, A. F. Análise de uma Unidade de Processamento de Roupas de um Hospital Através da Simulação a Eventos Discretos. Produto \& Produção, v.13, p. 7-24, 2012.

MIRANDA, R. C.; MONTEVECHI, J. A. B.; SILVA, A. F.; MARINS, F. A. S. A new approach to reducing search space and increasing efficiency in simulation optimization problems via the Fuzzy-DEA-BCC. Mathematical Problems in Engineering, p.1-15, 2014. https://doi.org/10.1155/2014/450367

Revista Produção Online, Florianópolis, SC, v. 16, n. 3, p. 1058-1078, jul./set. 2016. 
NUTONIAN. Disponível em: http://www.nutonian.com/products/eureqa-desktop/register/. Acessado em: 19 de janeiro de 2016.

O'REILLY, U. M. Genetic programming: a tutorial introduction. In: 2014 conference companion on Genetic and evolutionary computation companion, Proceedings..., New York, NY, USA, p. 227-250, 2014.

ÓLAFSSON, S.; KIM, J. Simulation Optimization. In: Winter Simulation Conference, Proceedings... San Diego, CA, USA, 2002.

OLIVEIRA, R. B. M.; CORRÊA, V. A.; PATROCíNIO, L. E. N. Mapeamento do fluxo de valor em um modelo de simulação computacional. Revista Produção Online, v. 14, n. 3, p. 837861, 2014. https://doi.org/10.14488/1676-1901.v14i3.1461

PERGHER, I.; SILVA, L. A.; PACHECO, D. A. J.; VACCARO, G. L. R Análise do impacto da variabilidade de fluxo no dimensionamento de kanbans. Revista Produção Online, v. 14, n. 1, p. 115-142, 2014. https://doi.org/10.14488/1676-1901.v14.11.1542

QIU, H.; XU, Y.; GAO, L.; LI, X.; CHI, L. Multi-stage design space reduction and metamodeling optimization method based on self-organizing maps and fuzzy clustering. Expert Systems with Applications, v. 46, p. 180-195, 2016. https://doi.org/10.1016/i.eswa.2015.10.033

ROSEN, S. L.; HARMONOSKY, C. H.; TRABAND, M. T. Optimization of Systems with Multiple Performance Measures via Simulation: Survey and Recommendations. Computers and Industrial Engineering, v.54, p.327-339, 2007. https://doi.org/10.1016/j.cie.2007.07.004

ROSS, P. J. Taguchi techniques for quality engineering. New York: McGraw-Hill,

RYAN, J.; HEAVEY, C. Process modeling for simulation. Computers in Industry, v.57, n.5, p.437-450, 2006. https://doi.org/10.1016/j.compind.2006.02.002

SAGDEEV, D. I.; FOMINA, M. G.; MUKHAMEDZYANOV, G. K.; ABDULAGATOV, I. M. Experimental Study and Correlation Models of the Density and Viscosity of 1-Hexene and 1Heptene at Temperatures from (298 to 473) K and Pressures up to $245 \mathrm{MPa}$. Journal of Chemical \& Engineering Data, v. 59, n. 4, p. 1105-1119, 2014 https://doi.org/10.1021/je401015e

SAKTI, A.; MICHALEK, J. J.; FUCHS, E. R.; WHITACRE, J. F. A techno-economic analysis and optimization of $\mathrm{Li}$-ion batteries for light-duty passenger vehicle electrification. Journal of Power Sources, v. 273, p. 966-980, 2015. https://doi.org/10.1016/.jpowsour.2014.09.078

SARGENT, R. G. Verification and validation of simulation models. Journal of Simulation, v.7, n.1, p.12-24, 2013. https://doi.org/10.1057/jos.2012.20

SHAN, S.; WANG, G. G. Metamodeling for high dimensional simulation-based design problems. Journal of Mechanical Design, v. 132, n.5, p.01-36, 2010.

https://doi.org/10.1115/1.4001597

SHARDA, B.; BURY, S.B. Best practices for effective application of discrete event simulation in the process industries. In: Winter Simulation Conference, Proceedings... Phoenix, AZ, USA, 2011. https://doi.org/10.1109/WSC.2011.6147942

SHEN, H. WAN, H. Controlled sequential factorial design for simulation factor screening. Revista Produção Online, Florianópolis, SC, v. 16, n. 3, p. 1058-1078, jul./set. 2016. 
European Journal of Operational Research, v.198, p.511-519, 2009.

https://doi.org/10.1016/i.ejor.2008.09.005

SIEBERS, P.O.; MACAL, C.M.; GARNETT, J.; BUXTON, D.; PIDD, M. (2010). Discreteevent simulation is dead, long life agent-based simulation! Journal of Simulation, v.4, p. 204-210, 2010. https://doi.org/10.1057/jos.2010.14

SREČEC, S.; ČEH, B., CILER, T. S.; RUS, A. F. Empiric mathematical model for predicting the content of alpha-acids in hop (Humulus lupulus L.) cv. Aurora. SpringerPlus, v. 2, n. 1, p. 1-5, 2013. https://doi.org/10.1186/2193-1801-2-59

STEPONAVIČĖ, I.; RUUSKA, S.; MIETTINEN, K. A solution process for simulation-based multiobjective design optimization with an application in the paper industry. Computer - Aided Design, v.47, p.45-58, 2014. https://doi.org/10.1016/j.cad.2013.08.045

SWISHER, J. R.; HYDEN P. D.; JACOBSON, S. H.; SCHRUBEN, L.W. A Survey of Simulation Optimization Techniques and Procedures. In: Winter Simulation Conference, Proceedings... Orlando, FL, USA, 2000. https://doi.org/10.1109/WSC.2000.899706

TAGUCHI, G.; CHOWDHURY, S.; WU, Y. Taguchi's quality engineering handbook. New Jersey: John Wiley \& Sons, Inc., 2005.

VILLARREAL-MARROQUIN, M. G.; CASTRO, J. M.; CHACÓN-MONDRAGÓN, Ó. L.; CABRERA-RÍOS, M. Optimisation via simulation: a metamodelling-based method and a case study. European Journal of Industrial Engineering, v. 7, n. 3, p. 275-294, 2013. https://doi.org/10.1504/EJIE.2013.054131

WANG, S.; SCHONFELD, P. Genetic Algorithm for Selecting and Scheduling Waterway Projects. In: Nets Symposium, Proceedings... Salt Lake City, USA, 2006.

WILLIS, K.O.; JONES, D.F. Multi-objective simulation optimization through search heuristics and relational database analysis. Decision Support Systems, v.46, p.277-286, 2008.

https://doi.org/10.1016/j.dss.2008.06.012

ZEINALI, F.; MAHOOTCHI, M.; SEPEHRI, M. M. Resource planning in the emergency departments: A simulation-based metamodeling approach. Simulation Modelling Practice and Theory, v.53, p.123-138, 2015. https://doi.org/10.1016/j.simpat.2015.02.002

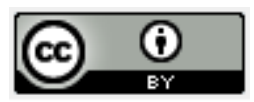

Artigo recebido em 21/01/2016 e aceito para publicação em 24/03/2016

DOI: http://dx.doi.org/ 10.14488/1676-1901.v16i3.2325 